“C 2011 IEEE. Personal use of this material is permitted. Permission from IEEE must be obtained for all other uses, in any current or future media, including reprinting/republishing this material for advertising or promotional purposes, creating new collective works, for resale or redistribution to servers or lists, or reuse of any copyrighted component of this work in other works." 


\title{
Performance Characteristics of an HTS Linear Synchronous Motor with HTS Bulk Magnets Secondary
}

\author{
Jianxun Jin, Luhai Zheng, Youguang Guo, Jianguo Zhu
}

\begin{abstract}
A single-sided high temperature superconducting (HTS) linear synchronous motor (HTSLSM) with HTS bulk magnet array as its secondary has been developed, and a split pulse coil magnetization system is used to obtain the secondary HTS bulk magnet array with alternating magnetic poles. The electromagnetic parameters of the HTSLSM are calculated to reflect its performance. A control system based on voltage space vector pulse width modulation (SVPWM) strategy implemented by the LabVIEW ${ }^{\mathrm{TM}}$ software controlled platform is developed to control the HTSLSM. An experimental system is also developed to measure the thrust and normal force of the HTSLSM. By plenty of investigations, the traits of the thrust and normal force have been found out, including the trends versus different exciting currents, different air gap lengths, variable magnetic poles, and different magnet arrangements. Some valuable conclusions are drawn and could benefit to the electromagnetic design and control scheme evaluation for the HTSLSM.
\end{abstract}

Keywords - High temperature superconductor (HTS), HTS linear synchronous motor (HTSLSM), HTS bulk magnet, split pulse coil, thrust and normal force.

\section{INTRODUCTION}

The high temperature superconducting (HTS) linear motors developed so far can be mainly classified by the principle and structure as follows: 1) HTS linear reluctance motor (LRM) with ordinary copper windings on the primary (stator) and zero-field-cooled (ZFC) HTS bulk poles on the secondary (mover) [1,2]; 2) HTS linear synchronous motor (HTSLSM) with ordinary copper windings on the primary and HTS bulk or coil magnets on the secondary [3-10]; 3) HTSLSM and linear induction motor (LIM) with HTS windings on the primary [11,12]. These HTS linear motors can be further divided into single-sided, double-sided, and cylindrical types according to their structures. Compared to the conventional linear motors, the HTS linear motors have a number of advantages, such as small size, low weight, and high efficiency because of the zero-resistance HTS tapes or strong-pinning HTS bulks with high trapped magnetic fields.

In this paper, a single-sided HTSLSM prototype with

This work was supported by the Chinese High-Tech R\&D (863) Program under Grant No. 2007AA03Z208.

Jianxun Jin and L. H. Zheng are with School of Automation, University of Electronic Science and Technology of China, Chengdu, China (e-mail: jxjin@uestc.edu.cn, zluhai@uestc.edu.cn).

Y. G. Guo, and J. G. Zhu are with Faculty of Engineering and Information Technology, University of Technology Sydney, Sydney, Australia (e-mail: youguang@eng.uts.edu.au, joe@eng.uts.edu.au).
HTS bulk magnet array as the secondary has been developed, and then a split pulse coil magnetization system is developed to obtain the secondary HTS bulk magnet array with alternating magnetic poles. The voltage space vector pulse width modulation (SVPWM) control strategy is used to the HTSLSM control system, and which is realized by LabVIEW ${ }^{\mathrm{TM}}$ software controlled platform. In order to clarify the dynamic performances of the HTSLSM, a testing system has been developed to study the thrust and normal force characteristics of the HTSLSM, mainly about their variations versus the exciting currents, the length of air gap, the number of magnetic poles and the magnet arrangements.

\section{Model AND Magnetization System OF HTSLSM}

\section{A. Model of HTSLSM}

The model of the developed single-sided HTSLSM is shown in Fig. 1. Its primary has a tooth-slot structure with concentrated winding, and the secondary is made of 24 YBCO HTS bulk magnets installed in a cryogenic vessel to form 6 magnetic poles with alternating poles ( $\mathrm{N}$ or $\mathrm{S}$ pole) along the moving direction (y-direction), and their same poles are installed sided by sided along transversal direction (z-direction) so to reach a suitable width as shown in Fig. 1(b) from the top view. Main dimensions are summarized in Table I.

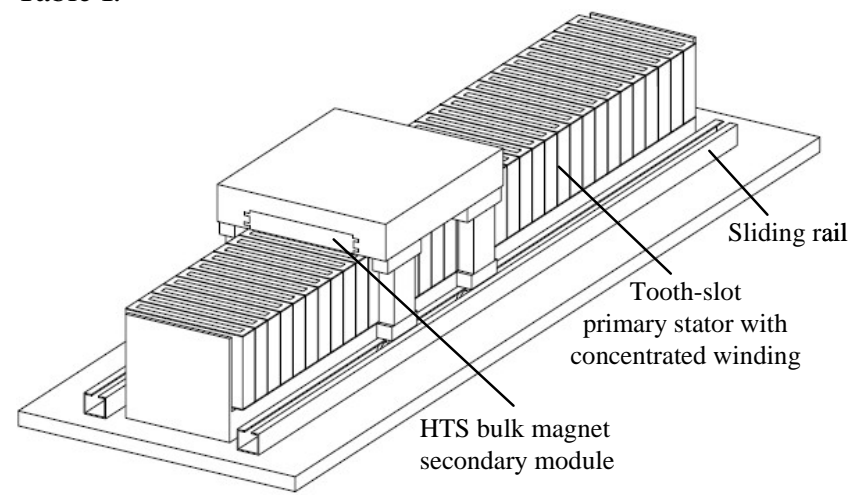

(a)

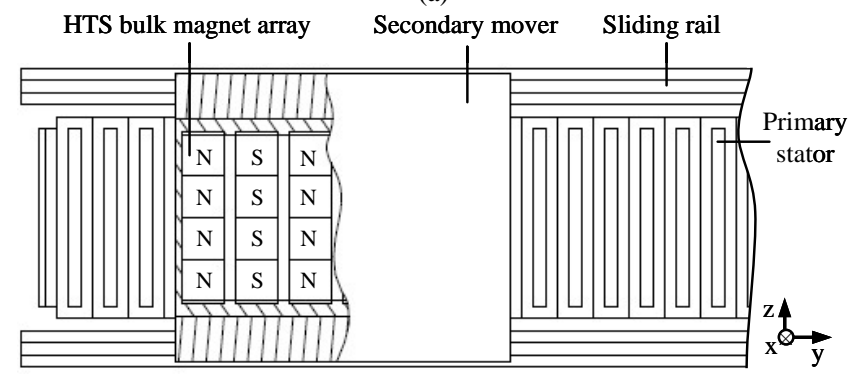

(b)

Fig. 1. Physical model of the HTSLSM, (a) 3D model, (b) Top view. TABLE I. Dimensions of the single-sided HTSLSM. (tooth width is 15 


\begin{tabular}{lll}
\hline mm) & & \\
& Slot width $w_{\mathrm{sl}}$ & $20 \mathrm{~mm}$ \\
& Slot depth $h_{\mathrm{sl}}$ & $100 \mathrm{~mm}$ \\
\multirow{3}{*}{ Primary } & Tooth length $l_{\mathrm{t}}$ & $10 \mathrm{~mm}$ \\
& Tooth width $w_{\mathrm{t}}$ & $15 \mathrm{~mm}$ \\
& Tooth pitch $y_{1}$ & $30 \mathrm{~mm}$ \\
& Pole pitch $\tau$ & $45 \mathrm{~mm}$ \\
\cline { 2 - 3 } & Turns of one phase winding $N_{1}$ & 200 \\
\hline \multirow{2}{*}{ Secondary } & Length $l_{\mathrm{s}}$ & $35 \mathrm{~mm}$ \\
mTS bulk & Width $w_{\mathrm{s}}$ & $35 \mathrm{~mm}$ \\
& Trapped $h_{\mathrm{s}}$ & $15 \mathrm{~mm}$ \\
& Relative permeability $\mu_{\mathrm{r}}$ & $0.5 \mathrm{~T}$ \\
& Number along $y$ direction & 0.4 \\
\hline \hline
\end{tabular}

\section{B. Magnetization system}

To realize alternating magnetic pole magnetization for the secondary HTS bulk magnets array, a split pulse coil magnetization system is proposed as shown in Fig. 2. After having magnetized one magnetic pole, we move the secondary module toward with a step length of one pole pitch $\tau$, and then magnetize the next HTS bulk in turn with an opposite magnetization direction, so as to obtain the alternating magnetic poles.

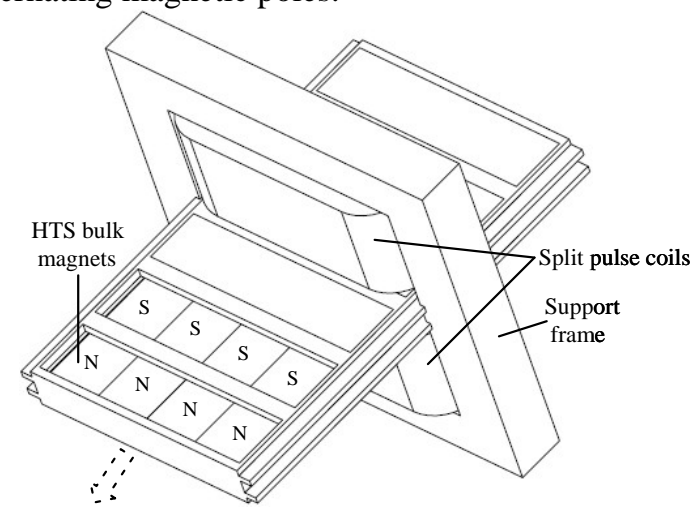

Fig. 2. Scheme of a split pulse coil system for magnetization of the HTS bulk magnet array having alternating magnetic poles.

The traits of trapping flux density $B_{\text {trap }}$ by pulse magnetization for two HTS bulk samples (BU1, BU2) having different qualities are measured and compared as shown in Fig. 3. It can be seen that the $B_{\text {trap }}$ of HTS bulk increases linearly with pulse field $B_{\text {pulse }}$ before saturation, where it has an optimum $B_{\text {pulse }}$ for saturation magnetization with maximum trapped magnetic field. The quality of HTS bulk also has substantial influence on the magnetic flux trapping capability. Therefore, an optimum $B_{\text {pulse }}$ can be found to magnetize the HTS bulks.

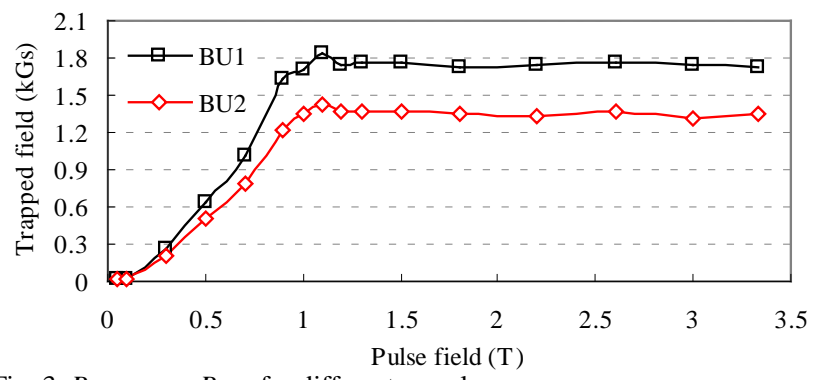

Fig. 3. $B_{\text {trap }}$ versus $B_{\text {pulse }}$ for different samples.
After having been magnetized, the magnetic flux density distribution of the HTS bulk magnet array with six magnetic poles is shown in Fig. 4, and the values of which are observed from its surface with a distance of $3 \mathrm{~mm}$.

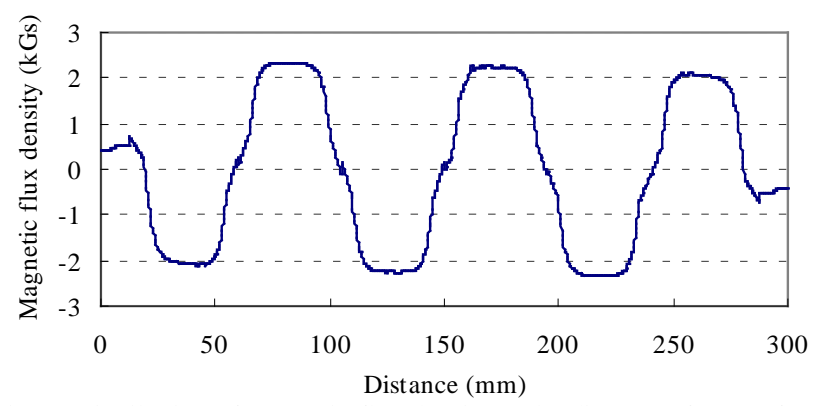

Fig. 4. Distribution of magnetic flux density at the distance of $3 \mathrm{~mm}$ from the surface of HTS bulk magnet array.

\section{PARAMETERS OF THE HTSLSM}

\section{A. Flux linkage}

The longitudinal section of the HTSLSM is shown in Fig. 5. Neglecting the magnetic resistance of primary iron-core, the magnetic flux linkage of HTS bulk magnets can be obtained approximately as

$$
\begin{gathered}
\psi_{\mathrm{SC}_{-} 1}=\frac{\mathscr{F}}{R_{\mathrm{tot}}}=\frac{2 P H_{\mathrm{sc}} \cdot h_{\mathrm{s}}}{\frac{2 g}{\mu_{\mathrm{r}} \mu_{0} l_{\mathrm{s}} w_{\mathrm{s}}}+\frac{2 g}{\mu_{0}\left(l_{\mathrm{s}}+l_{\mathrm{t}}\right)\left(w_{\mathrm{s}}+w_{\mathrm{t}}\right) / 4}+\frac{2 \tau}{\mu_{0} \cdot w_{\mathrm{s}} \cdot h_{\mathrm{s}}}(1)} \\
=\frac{P \cdot B_{\text {trap }} \cdot h_{\mathrm{s}}\left(l_{\mathrm{s}}+l_{\mathrm{t}}\right) \cdot\left(w_{\mathrm{s}}+w_{\mathrm{t}}\right) l_{\mathrm{s}} w_{\mathrm{s}} h_{\mathrm{s}}}{h_{\mathrm{s}}\left(l_{\mathrm{s}}+l_{\mathrm{t}}\right)\left(w_{\mathrm{s}}+w_{\mathrm{t}}\right) h_{\mathrm{s}}+4 \mu_{\mathrm{r}} g l_{\mathrm{s}} w_{\mathrm{s}} h_{\mathrm{s}}+\mu_{\mathrm{r}} \tau l_{\mathrm{s}}\left(l_{\mathrm{s}}+l_{\mathrm{t}}\right)\left(w_{\mathrm{s}}+w_{\mathrm{t}}\right)}
\end{gathered}
$$

where $\mathscr{F}$ is the magnetomotive force of magnetic circuit, $R_{\text {tot }}$ the total magnetic reluctance, $R_{s}$ the magnetic reluctance of HTS bulk magnet, $R_{g}$ the magnetic reluctance of air-gap (Rs and $\mathrm{Rg}$ are not in the equation), $\mu_{0}$ the permeability of vacuum, $\mu_{r}$ the relative permeability of HTS bulk magnet, $H_{\mathrm{sc}}$ the magnetic field intensity of HTS bulk magnet, $B_{\text {trap }}$ the trapped flux density of HTS bulk magnet, $g$ the air gap length, $P$ the pole pairs of HTS bulk magnets, $l_{s}, w_{s}$, and $h_{s}$ are the length, width and height of HTS bulk magnet respectively, $l_{t}, w_{t}$ the length and width of stator-tooth respectively. When the parameters shown in Table I are substituted in (1), the $\psi_{\mathrm{SC}_{-} 1}$ can be calculated out with a value of $1.86 \mathrm{mWb}$, as a result that the total flux linkage $\psi_{\mathrm{sc}}\left(=N_{1} * \psi_{\mathrm{SC} \_1}\right)$ is $0.37 \mathrm{~Wb}$.

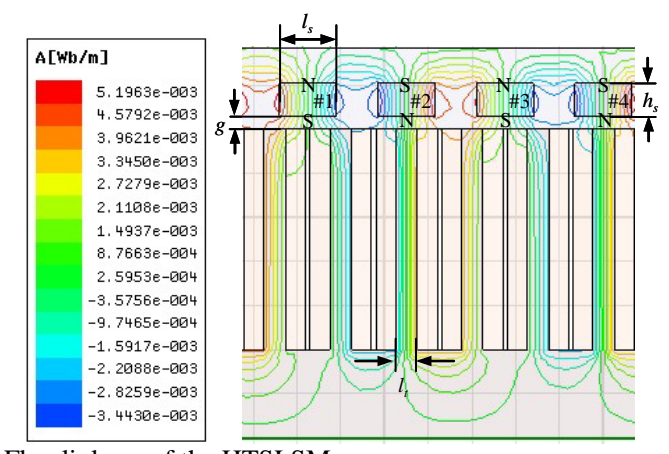

Fig. 5. Flux linkage of the HTSLSM.

The flux linkage also can be obtained by no-load finite 
element analysis (FEA) transient simulation based on Ansoft/Maxwell $^{\mathrm{TM}}$ software as shown in Fig. 6. It is observed that the flux waveform is an almost perfect sinusoid. The HTS bulk magnet flux waveforms are three phase symmetrical having the same magnitude of $0.33 \mathrm{~Wb}$ but are shifted by $120^{\circ}$ electrical to each other under the synchronous moving velocity $v_{\mathrm{s}}$ of $0.45 \mathrm{~m} / \mathrm{s}$. This is a fundamental requirement for three phase drive.

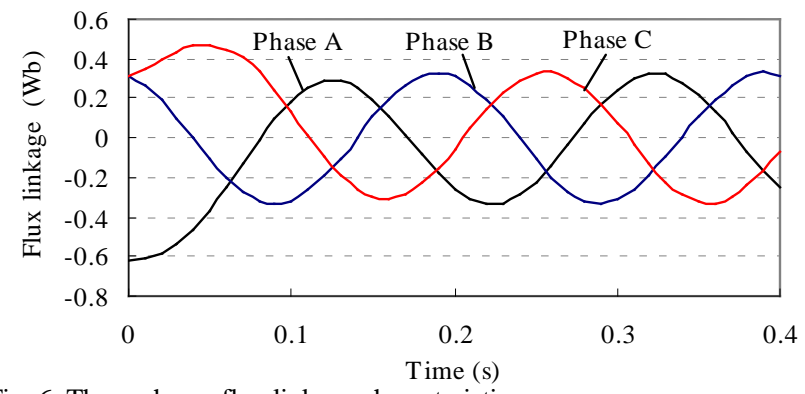

Fig. 6. Three-phases flux linkage characteristics.

\section{B. No-load Back EMF}

When the secondary rotor moves, a back electromotive force $(\mathrm{EMF}) E_{0}$ is induced in the stator windings. By differentiating the HTS bulk magnet flux of phase winding against time, the back EMF is determined with a root means square (RMS) value as

$$
E_{0}=\frac{1}{\sqrt{2}} \frac{\pi}{\tau} k_{N} \psi_{\mathrm{SC}} v_{s}=k_{E} v_{s}
$$

where $k_{E}=\pi k_{N} \psi_{\mathrm{SC}} /(\sqrt{2} \tau)$ is the back EMF constant, and $k_{\mathrm{N}}$ the winding factor, $v_{\mathrm{S}}$ the synchronous velocity, and $v_{\mathrm{s}}=2 \tau f$, $f$ the frequency. Based on the no-load transient simulation, the three-phases back EMF are obtained as shown in Fig. 7, which are sinusoid and symmetrical with the almost same amplitudes of $10.29 \mathrm{~V}$ under the $v_{\mathrm{s}}$ of 0.45 $\mathrm{m} / \mathrm{s}$.

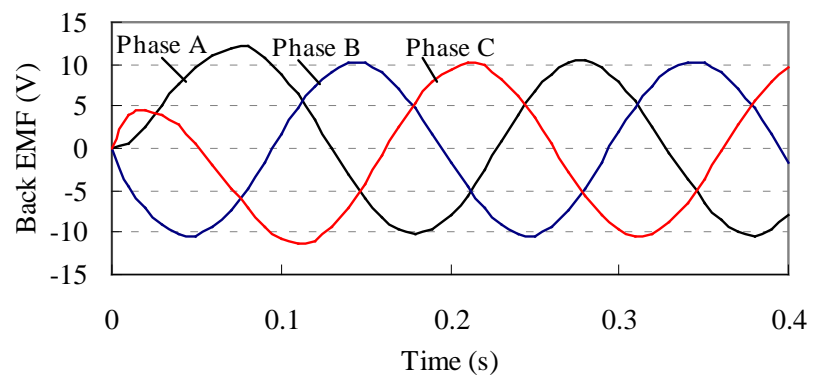

Fig. 7. Three-phases back EMF.

\section{C. $d$-axis and q-axis inductance}

Armature reaction inductance is [13]

$$
L_{a}=2 m \mu_{0} \frac{\left(N_{1} k_{N}\right)^{2}}{\pi^{2} P} \frac{w_{t}{ }^{\prime} \tau}{g^{\prime}}
$$

where $m$ is the number of phases, $g$ ' is the effective length of electromagnetic air gap, and $g^{\prime}=K_{c} g, K_{\mathrm{c}}$ the Carter coefficient, $w_{\mathrm{t}}$ ' the effective width of armature stator iron, and $w_{t}{ }^{\prime}=K_{E} w_{t}$, where $K_{\mathrm{E}}$ the angular coefficient.

The main leak inductance is

$$
L_{1}=2 m \mu_{0} w_{t}{ }^{\prime} \frac{\left(N_{1} k_{N}\right)^{2}}{\pi\left(P_{1}-P\right)}
$$

where $P_{1}$ is the total pole pairs of stator.

$\mathrm{d}$-axis synchronous inductance is

$$
L_{d}=K_{d} L_{m}+L_{1}=2 m \mu_{0}\left(N k_{N}\right)^{2} \frac{w_{t}{ }^{\prime}}{\pi}\left(\frac{\tau K_{d}}{\pi P \delta}+\frac{1}{P_{1}-P}\right)
$$

q-axis synchronous inductance is

$$
L_{q}=K_{q} L_{m}+L_{1}=2 m \mu_{0}\left(N k_{N}\right)^{2} \frac{w_{t}{ }^{\prime}}{\pi}\left(\frac{\tau K_{q}}{\pi P \delta}+\frac{1}{P_{1}-P}\right)
$$

where the $K_{\mathrm{d}}$ and $K_{\mathrm{q}}$ are the d-axis and q-axis armature reaction coefficients respectively.

The $L_{\mathrm{d}}$ and $L_{\mathrm{q}}$ are obtained by FEA transient simulation as indicated in Fig. 8. As shown, $L_{\mathrm{d}}$ is equal to $L_{\mathrm{q}}$ with a value of $0.381 \mathrm{H}$, when the magnetomotive force (MMF) is smaller than 2000 AN. After the MMF is bigger than the critical value, $L_{\mathrm{d}}$ and $L_{\mathrm{q}}$ decrease with the MMF, and the $L_{\mathrm{q}}$ becomes a bit smaller than the $L_{\mathrm{d}}$. This is mainly caused by the magnetic saturation of the primary stator iron.

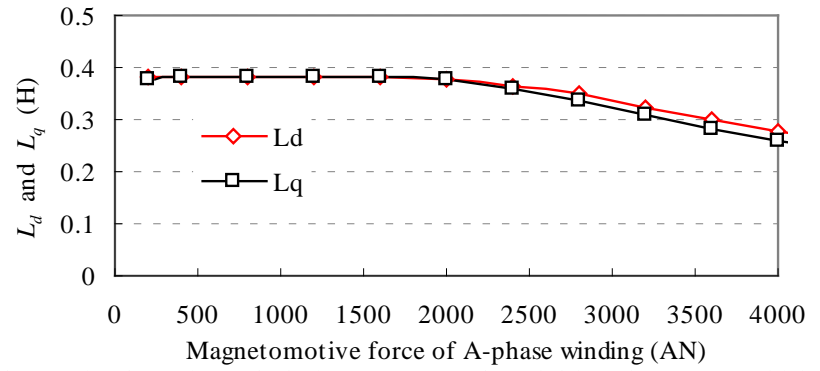

Fig. 8. d-axis and q-axis inductance. (x-axis subtitle: Phase A would be better than "A-phase")

\section{Synchronous reactance}

The armature reaction reactance is

$$
X_{\mathrm{a}}=2 \pi f L_{a}=4 m \mu_{0} f \frac{\left(N_{1} k_{\mathrm{N}}\right)^{2}}{\pi P} \frac{w_{t}{ }^{\prime} \tau}{g^{\prime}}
$$

The armature leakage reactance is

$$
X_{1}=4 m \mu_{0} f w_{t}{ }^{\prime} \frac{\left(N_{1} k_{\mathrm{N}}\right)^{2}}{P_{1}-P}
$$

The synchronous reactance is

$$
X_{t}=X_{a}+X_{1}
$$

\section{CONTROL System Design}

The voltage space vector pulse width modulation (SVPWM) strategy as an optimized PWM method is used to build up a simulation control platform by Matlab/Simulink $^{\mathrm{TM}}$ for the HTSLSM. Furthermore, SVPWM is realized by LabVIEW ${ }^{\mathrm{TM}}$ for the motor control practically.

\section{A. Closed Loop Control System for the HTSLSM}

In order to achieve the decoupling of the coupled equations, the mathematical model of HTSLSM is established under $d-q$ axis coordinate system as follows. 
Current equation

$$
\left[\begin{array}{c}
I_{d} \\
I_{q}
\end{array}\right]=\left[\begin{array}{cc}
-R / L_{d} & L_{q} \omega / L_{d} \\
-L_{d} \omega / L_{q} & -r / L_{q}
\end{array}\right]\left[\begin{array}{c}
I_{d} \\
I_{q}
\end{array}\right]+\left[\begin{array}{c}
U_{d} / L_{d} \\
\left(U_{q}-\psi_{\mathrm{sc}} \omega\right) / L_{q}
\end{array}\right]
$$

Electromagnetic thrust equation

$$
F_{\mathrm{em}}=\frac{3 P \pi}{2 \tau}\left[\psi_{\mathrm{sc}} I_{q}+\left(L_{d}-L_{q}\right) I_{q} I_{d}\right]
$$

Mechanical movement equation

$$
F_{\text {em }}=F_{L}+B_{v} v_{s}+M p v_{s}
$$

where $I_{\mathrm{d}}$ and $I_{\mathrm{q}}$ are d-axis and q-axis current, respectively, $r$ the resistance of a phase winding, $\omega$ the angular velocity of rotating machine converted from translatory velocity, $\omega=$ $\pi v_{\mathrm{s}} / \tau, U_{\mathrm{d}}$ and $U_{\mathrm{q}}$ the d-axis and q-axis voltage, respectively, $F_{\mathrm{L}}$ the load force, $B_{v}$ the mechanical damping coefficient relevant to velocity, $M$ the mass of motion parts, $p$ the differential operator, $p=\mathrm{d} / \mathrm{d} t$, and $R$ the resistance of phase winding.

Based on Eqs. (4)-(7), the simulation model of the HTSLSM is built up as shown in Fig. 9. Fig. 10 shows the closed loop speed-adjusting system simulation model for the HTSLSM based on SVPWM, and the SVPWM module as shown in Fig. 11. Fig. 12 shows the speed versus time characteristics, which indicates that the speed can reach a constant value in a short time.

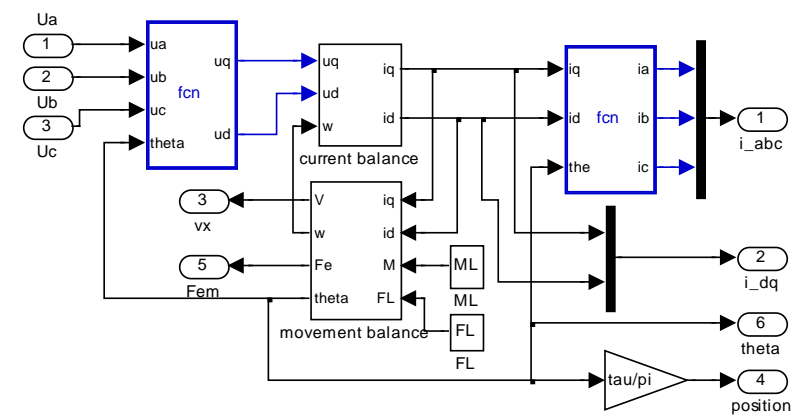

Fig. 9. Simulation model of HTSLSM.

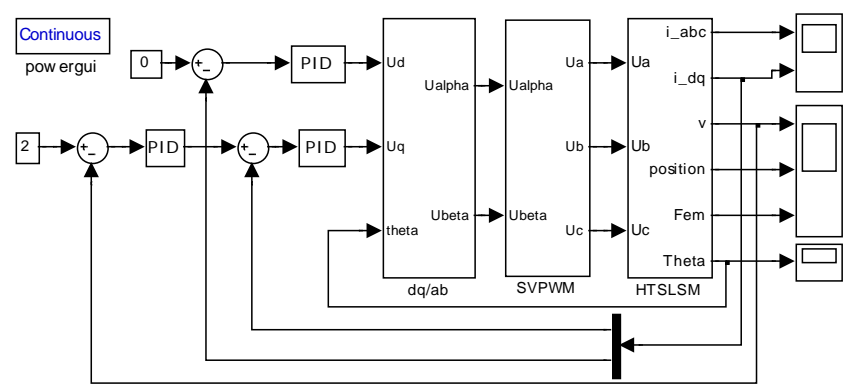

Fig. 10. Closed loop control system based on SVPWM for HTSLSM.

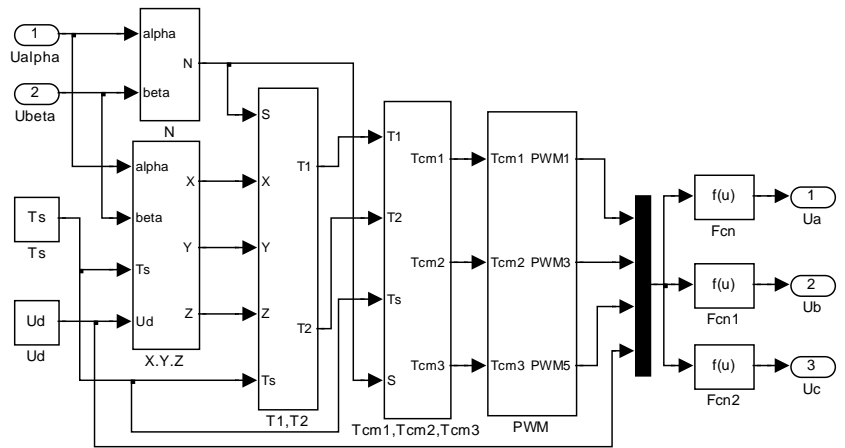

Fig. 11. Simulation model of SVPWM.

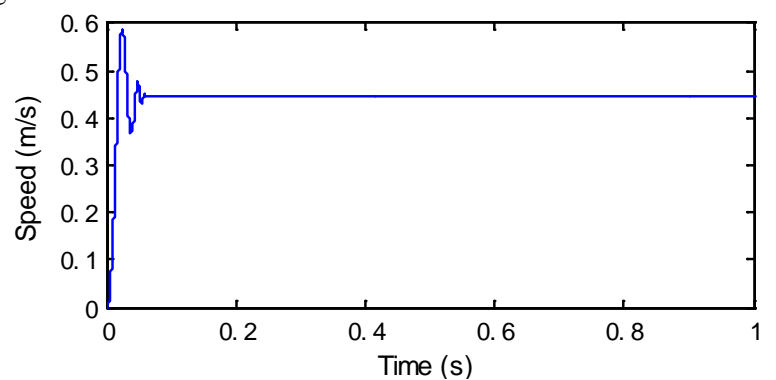

Fig. 12. Speed versus time characteristics.

\section{B. Realizing SVPWM by LabVIEW $W^{T M}$}

Based on the principle of SVPWM, the SVPWM signals are generated by the LabVIEW ${ }^{\mathrm{TM}}$ software with whose whole program block diagram as shown in Fig. 13. The SVPWM VI (Virtual instrument) is composed of some sub-VIs as follows: "Sector judgment.vi", "Choice of adjacent space voltage vectors.vi", "Calculation of operating time of basic vector.vi", "Sequence and time arrangement of basic vector.vi”, "Dead time inserting.vi" and "digital ouput.vi" etc.

Different to the Simulink ${ }^{\mathrm{TM}}$ simulation model of the HTSLSM speed-adjust system, the dead-time $t_{\mathrm{d}}$ must be inserted to the output sequence of the SVPWM practically produced by LabVIEW ${ }^{\mathrm{TM}}$, in order to avoid short circuit occurring. The block diagram of the dead time inserting subVI is shown in Fig. 14, and the output SVPWM waves after being added dead time are shown in Fig. 15.

The experimental closed-loop control system based on the LabVIEW $^{\mathrm{TM}}$ software controlled platform with SVPWM strategy is built up as shown in Fig. 16. Using this platform, the exciting current, various force signals like thrust and normal force can be acquired synchronously.

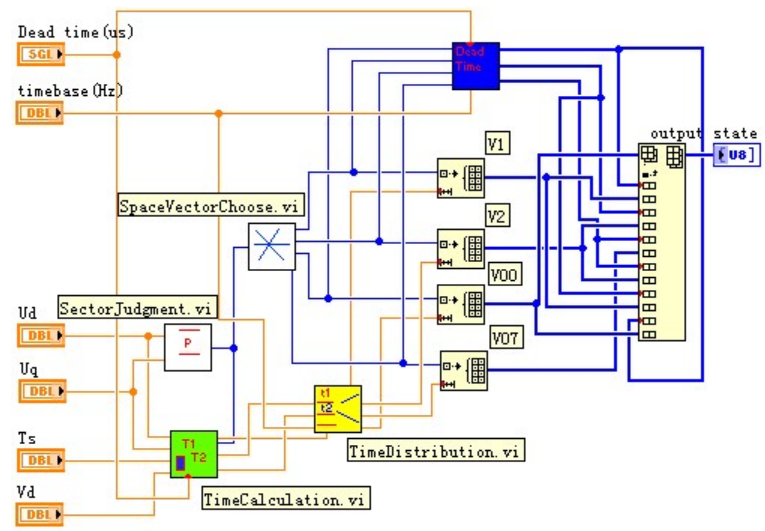

Fig. 13. The whole block diagram of SVPWM

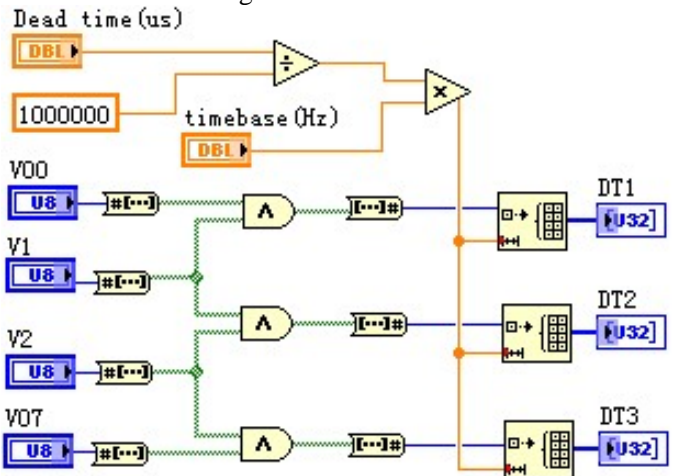


Fig. 14. Dead time inserting subVI.

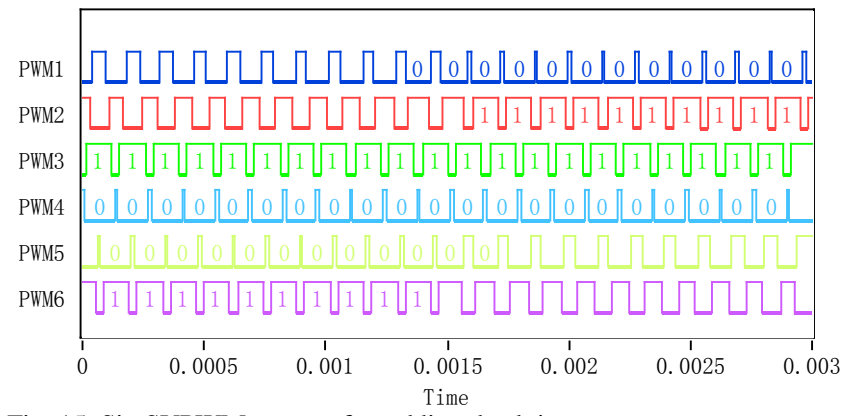

Fig. 15. Six SVPWM waves after adding dead time.

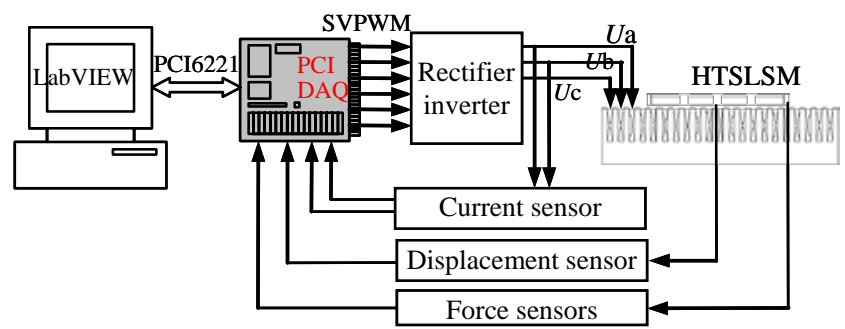

Fig. 16. LabVIEW based control and testing system for HTSLSM.

\section{Measurement System}

The measurement system for thrust and normal force of the HTSLSM is shown in Fig. 17. It mainly consists of one pulling sensor, four pressure sensors, one pulley, and a load. The locked-mover thrust is measured by using the pulling sensor connected to one side of the secondary mover, and the load connected to the other side of the mover. The normal force can be tested by using the four pressure sensors symmetrically fixed between the secondary mover and the wheels running along the tracks.

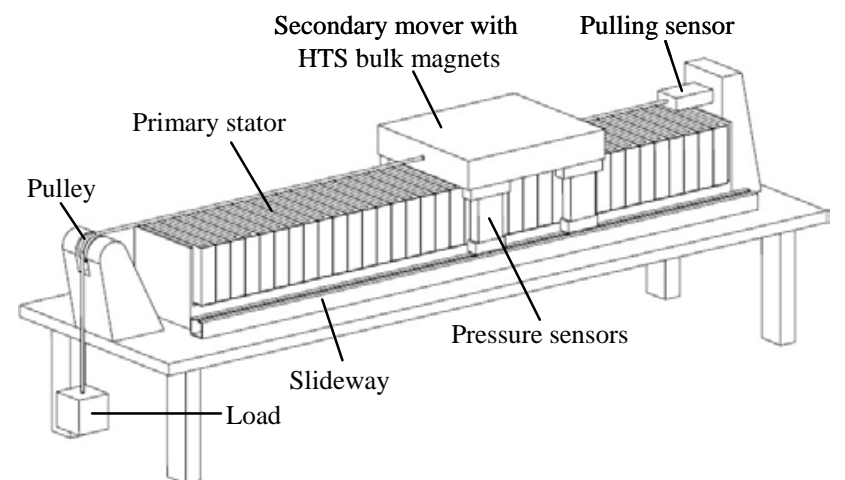

Fig. 17. Measurement system of thrust and normal force for the HTSLSM.

The standard secondary of the HTSLSM consists of 24 HTS bulk magnets with the same size of $35 \mathrm{~mm}$ (y-direction) * $37.5 \mathrm{~mm}$ (z-direction) $* 15 \mathrm{~mm}$ (x-direction) It composes 6 magnetic poles with $0.5 \mathrm{~T}$ trapped magnetic flux density.

The measured locked-mover thrust and normal force of the HTSLSM under the frequency of $12 \mathrm{~Hz}$, phase current amplitude of $3.36 \mathrm{~A}$, and air gap length of $10.5 \mathrm{~mm}$, are shown in Fig. 18. From Fig. 18(a), the waveform of the locked-mover thrust is nearly sinusoidal, and the amplitude of which is the maximum thrust $F_{\text {em_max }}$. The locked-mover normal force waveform from Fig. 18(b) is also nearly sinusoidal whose amplitude is the maximum normal force
$F_{\mathrm{n} \_ \text {max }}$. So the $F_{\mathrm{em} \_ \text {max }}$ and $F_{\mathrm{n} \_ \text {max }}$ of HTSLSM can be studied by measuring the amplitude of locked-mover thrust and normal force.

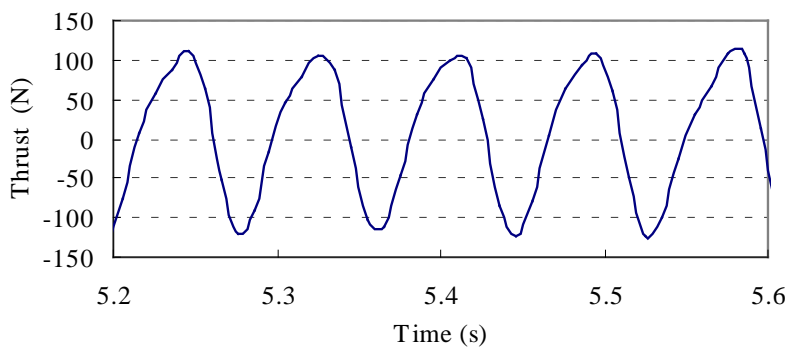

(a)

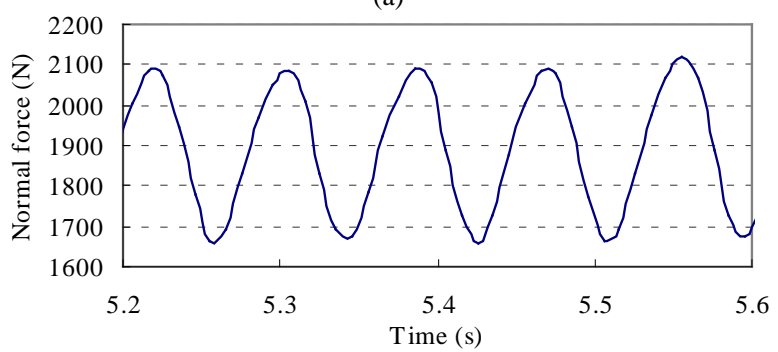

(b)

Fig. 18. Locked-mover thrust and normal force under $f=12 \mathrm{~Hz}, I=3.36 \mathrm{~A}$ $g=10.5 \mathrm{~mm}$. (a) $F_{\text {em }}$ versus time. (b) $F_{\mathrm{n}}$ versus time.

\section{RESUltS AND ANALYSIS}

\section{A. $F_{e m}$ and $F_{n}$ versus Exciting Current}

For the long-primary HTSLSM, the stator copper loss cannot be neglected [14], so the electromagnetic thrust can be derived as follows

$$
\begin{aligned}
F_{\text {em }} & =\frac{m U_{1} E_{0} \sqrt{X_{\mathrm{t}}^{2}+R_{1}^{2}} \sin (\theta+\phi)}{2 \tau f\left(X_{\mathrm{t}}^{2}+R_{1}^{2}\right)}-\frac{m E_{0}^{2} R_{1}}{2 \tau f\left(X_{\mathrm{t}}^{2}+R_{1}^{2}\right)} \\
& =\frac{m I_{1} E_{0}\left(X_{\mathrm{t}}+R_{1}\right) \sqrt{X_{\mathrm{t}}^{2}+R_{1}^{2}} \sin (\theta+\phi)-m E_{0}^{2} R_{1}}{2 \tau f\left(X_{\mathrm{t}}^{2}+R_{1}^{2}\right)}
\end{aligned}
$$

where $P_{\mathrm{em}}$ (not shown in the equation) is the electromagnetic power, $R_{1}$ the phase resistance, $U_{1}$ the phase voltage, and $U_{1}=I_{1}\left(X_{t}+R_{1}\right), I_{1}$ the phase current, $\phi$ the load shift angle, and $\phi=\arctan R_{1} / X_{\mathrm{t}}$. When load angle $\theta=90^{\circ}-\phi$, the maximum thrust $F_{\text {em_max }}$ can be obtained.

For the HTS bulk magnet, the fictitious equivalent current $I_{f}$ can be obtained corresponding to its coercive force $H_{\mathrm{sc}}$ and height $h_{\mathrm{s}}$,

$$
I_{f}=H_{\mathrm{sc}} h_{\mathrm{s}}
$$

The d-axis current and armature currents given by (The equation writes Iq) 


$$
\left\{\begin{array}{c}
I_{q}=\sqrt{\frac{2}{3}}\left[I_{a} \sin \theta+I_{b} \sin \left(\theta-\frac{2 \pi}{3}\right)+I_{c} \sin \left(\theta+\frac{2 \pi}{3}\right)\right] \\
I_{a}=\sqrt{2} I_{1} \cos \omega t \\
I_{b}=\sqrt{2} I_{1} \cos \left(\omega t-\frac{2 \pi}{3}\right) \\
I_{c}=\sqrt{2} I_{1} \cos \omega t\left(\omega t+\frac{2 \pi}{3}\right)
\end{array}\right.
$$

From (12), the q-axis current $I_{\mathrm{q}}$ is obtained as

$$
I_{q}=\sqrt{3} \sin \gamma_{0} \cdot I_{1}
$$

where $\gamma_{0}$ is electromagnetic force angle, which is the angle between the armature current $I_{1}$ and the HTS bulk magnet equivalent current $I_{f}$, and $\gamma_{0}=\omega t-\pi x / \tau$, where $x$ is the actual distance (the position of the mover) between axis of the magnet pole and axis of the phase A winding.

So the normal force equation is [13]

$$
F_{\mathrm{n}}=\frac{3}{2}\left[\left(I_{q}+I_{f}\right)^{2} \times \frac{L_{d}}{g}+I_{q}{ }^{2} \times \frac{L_{q}}{g}\right]
$$

When $B_{\text {trap }}=0.5 \mathrm{~T}, f=5 \mathrm{~Hz}, g=10.5 \mathrm{~mm}$, the $F_{\text {em_max }}$ is measured as a function of phase current $I_{1}$ as shown in Fig. 19. It can be seen that the $F_{\text {em_max }}$ increases almost linearly with $I_{1}$, and shows a good agreement with the calculated results by the magnetic circuit method. The $F_{\mathrm{n} \_ \text {max }}$ also increases linearly with the phase current.

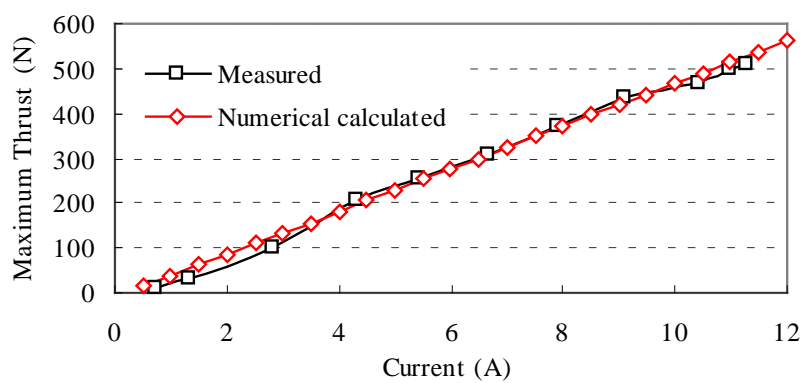

Fig. 19. $F_{\text {em_max }}$ versus phase current $(f=5 \mathrm{~Hz}, g=10.5 \mathrm{~mm})$.

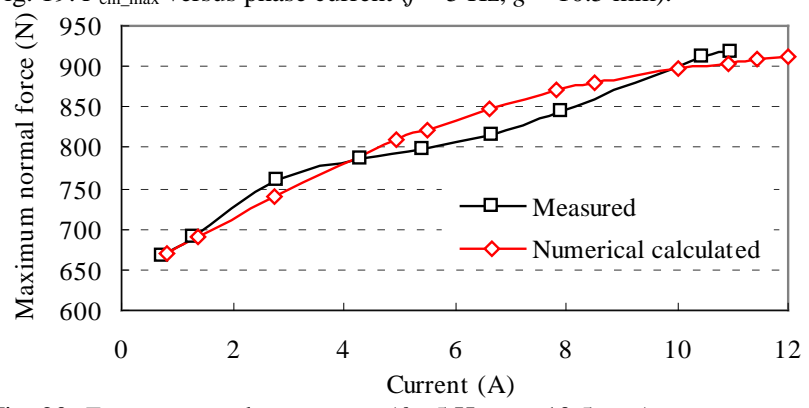

Fig .20. $F_{\mathrm{n} \_ \text {max }}$ versus phase current $(f=5 \mathrm{~Hz}, g=10.5 \mathrm{~mm})$.

\section{B. $F_{e m}$ and $F_{n}$ versus Length of Air Gap}

The influences of air gap length $g$ on the $F_{\text {em }}$ and $F_{\mathrm{n}}$ are investigated by changing the $g$ from $10.5 \mathrm{~mm}$ to $16 \mathrm{~mm}$. The measurements show that the $F_{\text {em_max }}$ and $F_{\text {n_max }}$ decrease linearly as $g$ increases as illustrated in Fig. 15. However, the slope of $F_{\mathrm{n} \_ \text {max }}$ decreasing rate is bigger than that of $F_{\text {em_max }}$ obviously. When $f=5 \mathrm{~Hz}, I=11.26 \mathrm{~A}$, the value of $F_{\mathrm{n} \_\max } / F_{\text {em_max }}$ changes from 1.8 to 1.3 as $g$ varies from 10.5 to $16 \mathrm{~mm}$ as shown in Fig. 16.

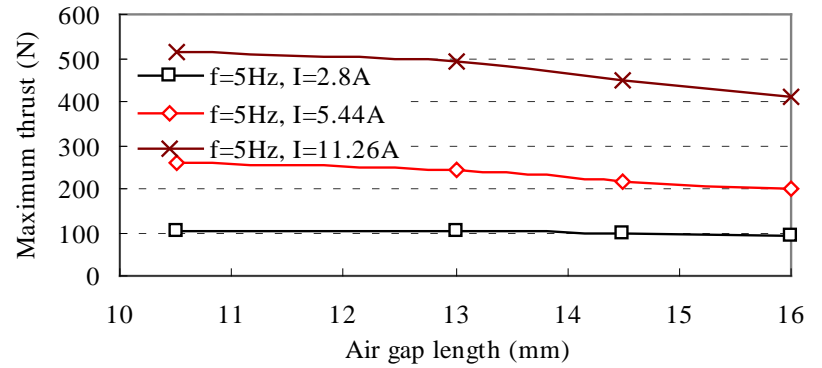

(a)

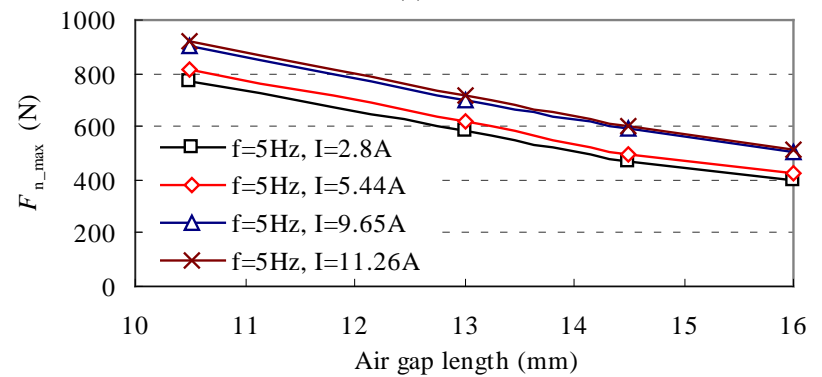

(b)

Fig. 15. $F_{\text {em_max }}$ and $F_{\text {n_max }}$ influenced by air gap length $g$ for various working current. (a) $F_{\text {em_max }}$ versus $g$. (b) $F_{\text {n_max }}$ versus $g$.

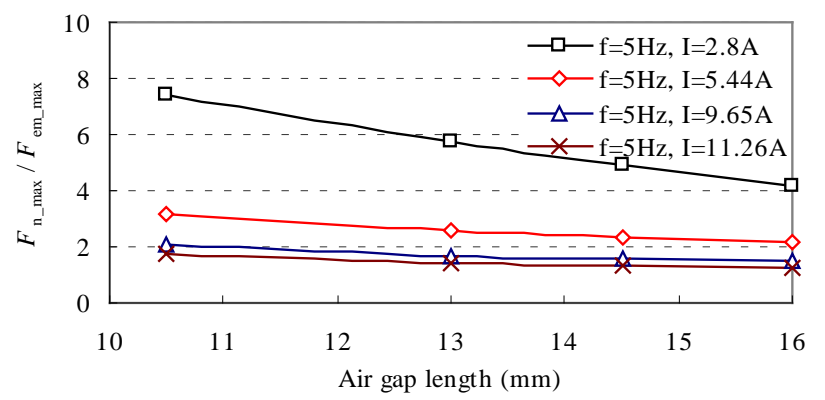

Fig. 16. $F_{\mathrm{n} \_ \text {max }} / F_{\text {em_max }}$ versus air gap length.

\section{C. $F_{e m}$ and $F_{n}$ versus the Number of Magnetic Poles}

When $g=13 \mathrm{~mm}$, the $F_{\text {em_max }}$ and $F_{\text {n_max }}$ are measured in case of that magnetic poles $n$ is 2, 4, and 6 respectively. The results indicate that $F_{\text {em_max }}$ and $F_{\text {n_max }}$ increase linearly with $n$ as shown in Fig. 17, and the slop of rising rates increases with the amplitude of phase current. When the $I$ is bigger than a critical value applied, the $F_{\mathrm{n} \_\max } / F_{\text {em_max }}$ could decrease linearly with $n$ as shown in Fig. 18. For example, if change $n$ from 2 to 6 under $I=11.26$ A, the ratio will decrease from 2.4 to 1.5 .

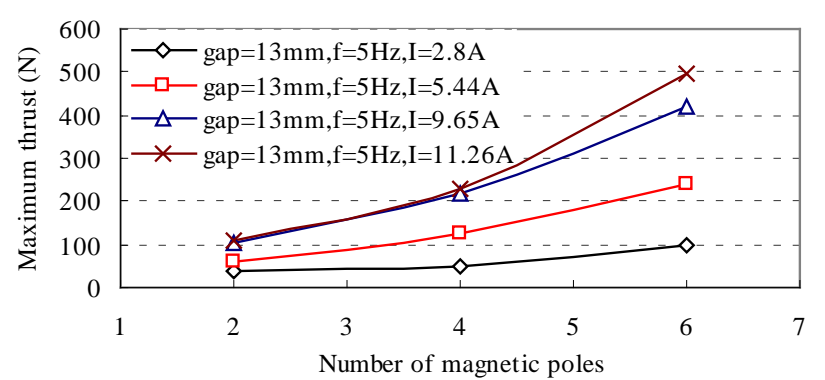

(a) 


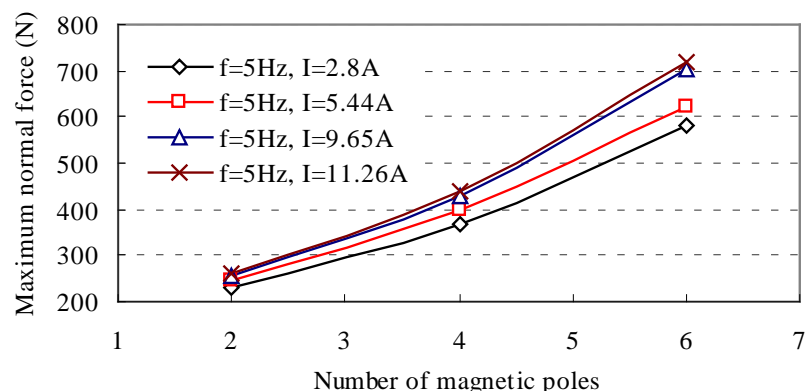

(b)

Fig. 17. $F_{\text {em_max }}$ and $F_{\text {n_max }}$ influenced by the number of magnetic poles $n$ for various working current. (a) $F_{\text {em_max }}$ versus $n$. (b) $F_{\mathrm{n} \_ \text {max }}$ versus $n$.

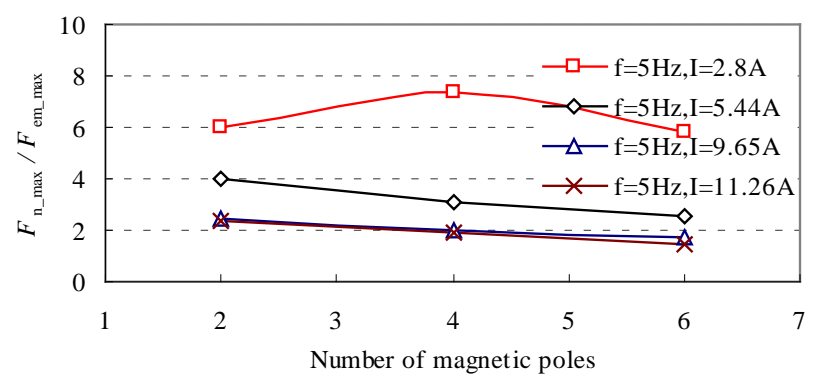

Fig. 18. $F_{\mathrm{n} \_ \text {max }} / F_{\text {em_max }}$ versus number of magnetic poles.

\section{D. $F_{\text {em }}$ and $F_{n}$ versus Different Magnet Arrangements}

The influences of different magnet arrangements on the $F_{\text {em }}$ and $F_{\mathrm{n}}$ are also studied experimentally. Fig. 19(a) and Fig. 19(b) show the secondary HTS bulk magnet arrays with total 16 bulk magnets arranged in the form of 4 $($ y-direction $) \times 4$ (x-direction) and 8 (y-direction) $\times 2$ (x-direction), respectively. The $F_{\mathrm{em} \text { max }}$ and $F_{\mathrm{n} \_ \text {max }}$ of the two secondary models as a function of phase current are measured as illustrated in Fig. 20. It is found out that the magnet array of $8 \times 2$ generates larger $F_{\text {em_max }}$ and $F_{\text {n_max }}$ with smaller ratio of $F_{\mathrm{n} \_ \text {max }} / F_{\text {em_max }}$ than that of the magnet array $4 \times 4$ as shown in Fig. 21 .

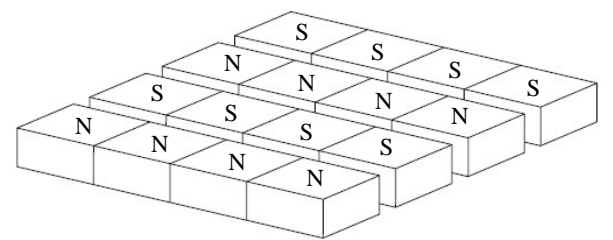

(a)

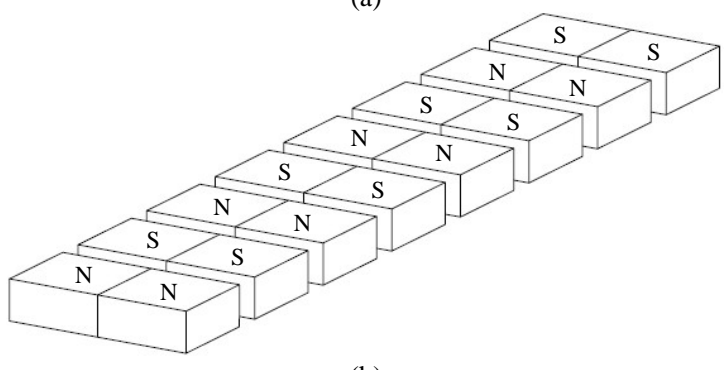

(b)

Fig. 19. Different magnet arrangements. (a) 4 (y-direction) $\times 4$ (x-direction). (b) 8 (y-direction) $\times 2$ (x-direction).

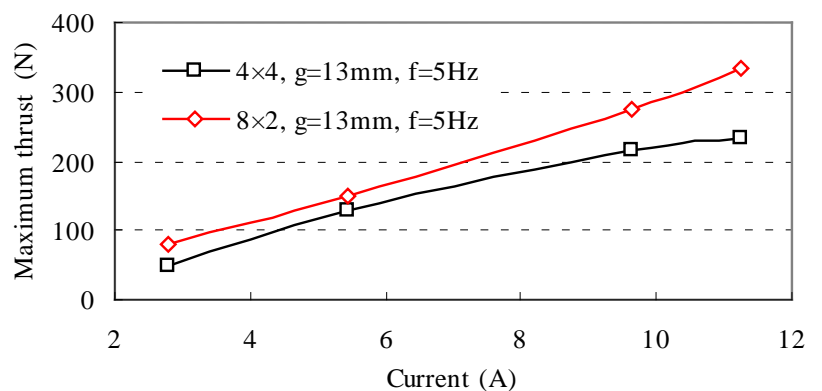

(a)

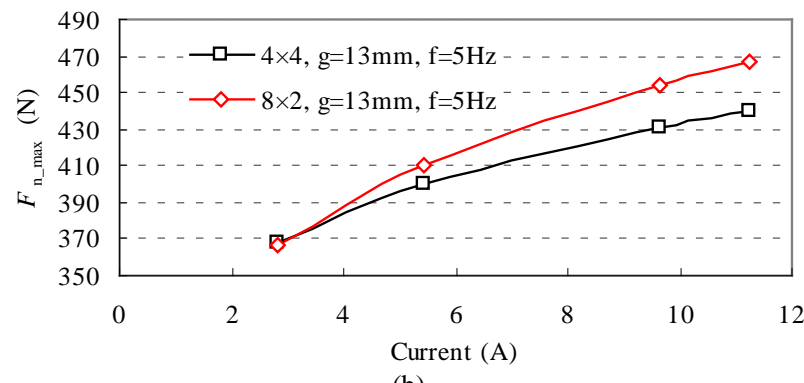

(b)

Fig. 20. $F_{\text {em_max }}$ and $F_{\text {n_max }}$ influenced by secondary magnet arrangements. (a) $F_{\text {em_max }}$ versus current for different magnet arrangements. (b) $F_{\mathrm{n} \_\max }$ versus current for different magnet arrangements.

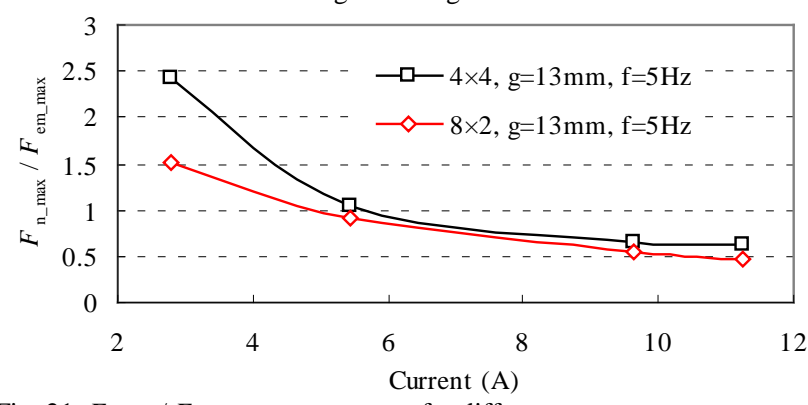

Fig. 21. $F_{\text {n_max }} / F_{\text {em_max }}$ versus current for different magnet arrangements.

\section{CONCLUSIONS}

A single-sided HTSLSM prototype with HTS bulk magnet array as the secondary has been developed with a split pulse coil magnetization system to obtain the secondary HTS bulk magnet array with alternating magnetic poles. The electromagnetic parameters of the motor were calculated by magnetic circuit calculation and FEA method to show its performance. The control system based on SVPWM implemented by the LabVIEW $^{\mathrm{TM}}$ software controlled platform was proposed and introduced. The thrust and normal force compositive measurement system for the HTSLSM was developed to measure the thrust and normal force, and the results show that the maximum thrust and normal force increase linearly by increasing the current and the number of magnetic poles, or decreasing the air gap length, while the ratio of $F_{\mathrm{n} \_\max } /$ $F_{\text {em_max }}$ decreases with the current, air gap length and number of magnetic poles. Under the same number of the total secondary magnets, the array with more poles has obtained a better drive performance. This work will benefit to the electromagnetic design of an HTSLSM especially to the secondary HTS bulk magnet array.

\section{REFERENCES}

[1] K. Yoshida, H. Matsumoto, and M. Eguchi, “Optimal design of thrust 
force in vertical-type HTS bulk LRM,” Physica C, vol. 426-431, no. 1, pp. 839-847, 2005.

[2] R. Muramatsu, S. Sadakata, M. Tsuda, and A. Ishiyama, "Trial production and experiments of linear actuator with HTS bulk secondary,” IEEE Trans. Appl. Superconduct., vol. 11, no. 1, pp. 1976-1979, 2001.

[3] G. Stumberger, M.T. Aydemir, and A.L. Thomas, "Design of a linear bulk superconductor magnet synchronous motor for electromagnetic aircraft launch systems," IEEE Trans. Appl. Superconduct., vol. 14, no. 1, pp. 54-62, 2004.

[4] L. H. Zheng, J. X. Jin, Y. G. Guo, H. Y. Lu, and J. G. Zhu, "Design and electromagnetic analysis of a HTS linear synchronous motor," in Proc. IEEE ASEMD, 2009, pp. 5-10.

[5] A. Takahashi, H. Ueda, and A. Ishiyama, "Trial production and experiment of linear synchronous actuator with field-cooled HTS bulk secondary,” IEEE Trans. Appl. Superconduct., vol. 13, no. 2, pp. 2251-2254, 2003.

[6] A. Sato, H. Ueda, and A. Ishiyama, "Operational characteristics of linear synchronous actuator with field-cooled HTS bulk secondary," IEEE Trans. Appl. Superconduct., vol. 15, no. 2, pp. 2234-2237, 2005.

[7] S. Kusada, M. Igarashi, K. Nemoto, T. Okutomi, S. Hirano, K. Kuwano, T. Tominaga, M. Terai, T. Kuriyama, K. Tasaki, T. Tosaka, K. Marukawa, S. Hanai, T. Yamashita, Y. Yanase, H. Nakao, and M. Yamaji, "The project overview of the HTS magnet for superconducting maglev,” IEEE Trans. Appl. Superconduct., vol. 17, no. 2, pp. 2111-2116, 2007.

[8] K. Kuwano, M. Igarashi, S. Kusada, K. Nemoto, T. Okutomi, S. Hirano, T. Tominaga, M. Terai, T. Kuriyama, K. Tasaki, T. Tosaka, K. Marukawa, S. Hanai, T. Yamashita, Y. Yanase, H. Nakao, and M. Yamaji, "The running tests of the superconducting maglev using the HTS magnet," IEEE Trans. Appl. Superconduct., vol. 17, no. 2, pp. 2125-2128, 2007.

[9] K. Tasaki, K. Marukawa, S. Hanai, T. Tosaka, T. Kuriyama, T. Yamashita, Y. Yanase, M. Yamaji, H. Nakao, M. Igarashi, S. Kusada, K. Nemoto, S. Hirano, K. Kuwano, T. Okutomi, and M. Terai, "HTS magnet for maglev applications (1) — coil characteristics," IEEE Trans. Appl. Superconduct., vol. 16, no. 2, pp. 1100-1103, 2006.

[10] M. Terai, M. Igarashi, S. Kusada, K. Nemoto, T. Kuriyama, S. Hanai, T. Yamashita, and H. Nakao, "The R\&D project of HTS magnets for the superconducting maglev," IEEE Trans. Appl. Superconduct., vol. 16, no. 2, pp. 1124-1129, 2006.

[11] W. S. Kim, S. Y. Jung, H. Y. Choi, H. K. Jung, J. H. Kim, and S. Y. Hahn, "Development of a superconducting linear synchronous motor," IEEE Trans. Appl. Superconduct., vol. 22, no. 1, pp. 842-845, 2002.

[12] T. Kikuma and A.Ishiyama, "Improvement of superconducting cylindrical linear induction motor," IEEE Trans. Appl. Superconduct., vol. 11, no. 1, pp. 2331-2334, 2001.

[13] G. Xiong and S. A. Nasar, "Analysis of fields and forces in a permanent magnet linear synchronous machine based on the concept of magnetic charge," IEEE Trans. Magn., vol. 25, no. 3, pp. 2713-2719, 1989.

[14] Z. Deng, I. Boldea, and S. Nasar, "Forces and parameters of permanent magnet linear synchronous machines,” IEEE Trans. Magn., vol. 23, no. 1, pp. 305-309, 1987.

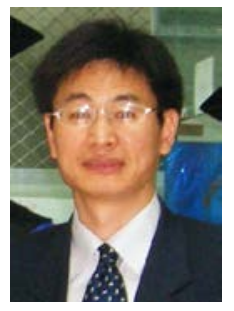

Jian Xun Jin, Professor, Ph.D., Ph.D. adviser, is currently the Director of the Center of Applied Superconductivity and Electrical Engineering at the University of Electronic Science and Technology of China. Research interests mainly include applied superconductivity, electromagnetic devices, electric machine, electric power, control, measurement and energy efficiency technology.

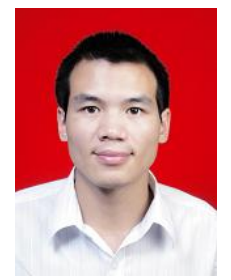

Lu Hai Zheng was born in Zhejiang, China in 1980. He received B.Eng. degree from Huaiyin Teachers College in 2005. He is currently pursuing the Ph.D. degree with University of Electronic Science and Technology of China. His research direction is high temperature superconducting linear machine technology.

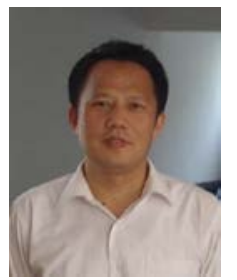

You Guang Guo (S'02-M'05-SM'06, IEEE) was born in Hubei, China in 1965 . He received the B.E. degree from Huazhong University of Science and Technology (HUST), China in 1985, the M.E. degree from Zhejiang University, China in 1988, and the Ph.D. degree from University of Technology, Sydney (UTS), Australia in 2004, all in electrical engineering. His research fields include measurement and modeling of magnetic properties of magnetic materials, numerical analysis of electromagnetic field, electrical machine design and optimization, power electronic drives and control.

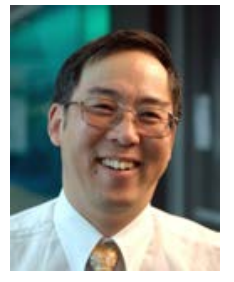

Jian Guo Zhu received his BE in 1982 from Jiangsu Institute of Technology, China, ME in 1987 from Shanghai University of Technology, China, and Ph.D. in 1995 from University of Technology, Sydney (UTS), Australia, He currently holds the positions of Professor of Electrical Engineering and Head for School of Electrical, Mechanical and Mechatronic Systems at UTS, Australia. His research interests include electromagnetics, magnetic properties of materials, electrical machines and drives, power electronics, and renewable energy systems. 